\section{Aquisição de açúcar e perfil de macronutrientes na cesta de alimentos adquirida pelas famílias brasileiras (2002-2003)}

\author{
Sugar and overall macronutrient profile in the \\ Brazilian family diet (2002-2003)
}

\author{
1 Faculdade de Medicina, \\ Universidade de São Paulo, \\ São Paulo, Brasil. \\ 2 Núcleo de Pesquisas \\ Epidemiológicas em Nutrição \\ e Saúde, Universidade de São \\ Paulo, São Paulo, Brasil. \\ 3 Faculdade de Saúde \\ Pública, Universidade de São \\ Paulo, São Paulo, Brasil. \\ Correspondência \\ R. B. Levy \\ Departamento de Medicina \\ Preventiva, Faculdade de \\ Medicina, Universidade de \\ São Paulo. \\ Av Dr. Arnaldo 455, 1o andar \\ São Paulo, SP 01246-903, \\ Brasil. \\ rlevy@usp.br
}

\begin{abstract}
This study evaluated the impact of calories from sugar on the calorie share of other macronutrients in the Brazilian family diet. The study's estimates are based on data from the Household Budget Survey conducted by the Brazilian Institute of Geography and Statistics from July 2002 to June 2003. Multiple linear regression models were used to study the impact of sugar calories on each of the macronutrients in household food acquisition, controlling for total energy content in food acquisition and socio-demographic variables. Each calorie from sugar purchases increases the share of calories from fat by 0.3 calories and decreases the share of calories from protein by 0.7 calories. Each calorie of sugar from processed foods increases the share from fat by 1.6 calories and the share from saturated fatty acids by $0.4 \mathrm{cal}$ ories and decreases the share from non-sugar carbohydrates by 0.8 calories. The findings shed further light on the harmful role of sugar for human health.
\end{abstract}

Feeding Behavior; Sugar; Eating
Renata Bertazzi Levy 1,2

Rafael Moreira Claro 2

Carlos Augusto Monteiro 2,3

\section{Introdução}

Evidências apontam para o aumento da prevalência de obesidade e sobrepeso, em paralelo à redução da desnutrição, ao redor do mundo 1 . Dados de inquéritos nacionais, de base populacional, realizados no Brasil entre 1975 e 2003 evidenciam declínio contínuo da prevalência de déficits ponderais e avanço alarmante da prevalência de excesso de peso e obesidade, em todas as regiões do país e em todas as classes de renda 2,3,4. A trajetória dos indicadores nutricionais descritos se deve a mudanças na composição das dietas e no estilo de vida dos indivíduos decorrentes de mudanças no ambiente relacionadas à industrialização, urbanização e integração daqueles países à economia global 5 .

Estudos sobre a evolução da disponibilidade de alimentos na América Latina indicam tendência de aumento na participação de gorduras na dieta, especialmente gordura de origem animal, e aumento da já elevada participação de calorias de açúcar. Entre 1970-1972 e 1995-1996, a participação do açúcar na disponibilidade de alimentos dos países latino-americanos aumentou de $15,8 \%$ para $16,9 \%$. No Brasil, a evolução da disponibilidade nacional de alimentos entre 19611963 e 1999-2001 aponta grande aumento na participação de gorduras (de 15,7\% para 26,3\% das calorias totais) acompanhada de aumento discreto na já elevada participação de açúcar (de $18 \%$ para $18,8 \%$ das calorias totais) ${ }^{4}$ (Food 
and Agriculture Organization. Statistic database. http:/ / faostat.fao.org/faostat/collections?versio $\mathrm{n}=$ ext\&hasbulk=0, acessado em Set/2007).

$\mathrm{O}$ acúmulo de evidências que indicam que determinadas características da dieta aumentam substancialmente o risco de doenças cardiovasculares, certos tipos de câncer, obesidade, dislipidemias e diabetes, entre outras, fez com que a Organização Mundial da Saúde (OMS) 8 estabelecesse limite máximo de consumo para a ingestão de gorduras (30\% do valor calórico total), ácidos graxos saturados ( $10 \%$ do consumo calórico total), colesterol (300mg/dia) e açúcar ( $10 \%$ do valor calórico total) e limites mínimos para o consumo de frutas e hortaliças $(400 \mathrm{~g} / \mathrm{dia})$ e carboidratos complexos (entre 55 e $75 \%$ do valor calórico total).

Embora a recomendação da OMS 7 em limitar o consumo de açúcar não seja seguida por todos os países - nos Estados Unidos e Canadá, por exemplo, o limite superior para a participação do açúcar nas calorias totais é de $25 \% 8$-, ela encontra suporte em vários estudos que associam o consumo de açúcar a efeitos desfavoráveis para a saúde. Dentre esses efeitos, destacam-se aumento na ocorrência de cárie dental 7 , ganho excessivo de peso ${ }^{9,10}$ e aumento na incidência de doenças cardiovasculares 11 e diabetes 12 .

Há também evidências de que o consumo de açúcar influencia negativamente características gerais da dieta, contribuindo para o consumo excessivo de calorias 13,14,15,16, diminuindo a participação de outros macronutrientes 14,17 e diluindo a concentração de micronutrientes 14,15,18.

No presente estudo será analisada a influência que a aquisição de calorias provenientes de açúcar (açúcar de mesa ou açúcar presente em alimentos processados) exerce sobre a contribuição calórica de cada um dos demais macronutrientes na aquisição domiciliar de alimentos.

\section{Métodos}

Os dados utilizados neste estudo são originários da Pesquisa de Orçamentos Familiares realizada pelo Instituto Brasileiro de Geografia e Estatística no período de junho de 2002 a julho de 2003 (POF 2002-2003), em uma amostra representativa de todos os domicílios do Brasil.

\section{Amostragem}

O desenho da amostra da POF 2002-2003 foi estruturado de modo a representar o conjunto dos domicílios brasileiros, bem como as cinco grandes regiões do país conforme situação urbana ou rural. Trata-se de uma amostra estra- tificada, sorteada em dois estágios. Os estratos, em número de 443, foram formados pelo agrupamento dos setores censitários homogêneos quanto ao domínio territorial (áreas urbanas ou rurais de um mesmo estado, segundo o Censo Demográfico de 2000; http://www.ibge.gov.br) e média de escolaridade do chefe do domicílio (obtida na Contagem Populacional de 1996). O sorteio de setores censitários (unidade primária de amostragem) dentro de cada estrato foi feito por amostragem sistemática com probabilidade proporcional ao número de domicílios no setor. O sorteio de domicílios (unidade secundária de amostragem) dentro de cada setor foi feito por amostragem aleatória simples, sem reposição. Para que a coleta de dados fosse uniforme nos quatro trimestres do ano, as entrevistas realizadas dentro de cada um dos 443 estratos foram distribuídas para estudo ao longo dos 12 meses de duração da pesquisa. No período, foram entrevistados 48.470 domicílios em 3.984 setores. Informações complementares ao detalhamento da amostra encontram-se disponíveis em publicação anterior ${ }^{2}$.

O período de referência utilizado pela POF 2002-2003 para a coleta de informações sobre aquisição de alimentos em cada domicílio é de apenas sete dias, o que não permite uma caracterização adequada do padrão de aquisição de alimentos da unidade domiciliar estudada. Por essa razão, na presente investigação, optou-se por adotar como unidade de estudo não o domicílio individual estudado pela pesquisa, mas o conjunto de domicílios existentes em cada um dos 443 estratos da amostra (média de 109,6 domicílios).

\section{Coleta de dados}

As informações dos alimentos adquiridos em cada domicílio dizem respeito ao registro de todas as aquisições de alimentos feitas durante um período de sete dias consecutivos, incluindo quantidade, unidade de medida com seu equivalente em peso ou volume, valor da despesa, local de compra e a forma de obtenção do alimento.

\section{Variáveis analisadas}

As variáveis utilizadas neste estudo são representadas pelas quantidades de açúcar, de outros carboidratos que não o açúcar, de proteínas e de gorduras presentes nas aquisições de alimentos feitas pelos domicílios que constituem cada estrato da amostra da POF 2002-2003. Tanto no caso do açúcar quanto no caso dos demais macronutrientes, as quantidades adquiridas pelo estrato são expressas em $\mathrm{kcal} /$ per capita/dia. No 
caso das calorias provenientes de açúcar foram consideradas tanto aquelas do açúcar de mesa quanto dos açúcares adicionados pela indústria aos alimentos. Rapadura, melado, mel e outros adoçantes calóricos, embora representem proporção diminuta do total de calorias adquiridas pelas famílias brasileiras, foram igualmente consideradas no cômputo do total de calorias provenientes de açúcar. Os açúcares intrínsecos, presentes naturalmente nos alimentos, como a frutose nas frutas e a lactose nos leites, foram considerados neste estudo como parte do restante dos carboidratos da alimentação. No caso das calorias provenientes de gorduras, foram consideradas separadamente as calorias provenientes de ácidos graxos saturados.

Os procedimentos empregados para se chegar à aquisição diária per capita de cada macronutriente (expressa em $\mathrm{kcal}$ ) em cada estrato de domicílios foram os seguintes: (1) soma das quantidades adquiridas de um mesmo alimento (em gramas) ao longo dos sete dias de coleta; (2) aplicação para cada alimento de fator de correção que leva em conta apenas as partes comestíveis dos alimentos 19; (3) conversão da quantidade adquirida de cada alimento em calorias de açúcar refinado e calorias dos demais macronutrientes de interesse, empregando-se para tanto a tabela TACO (Tabela Brasileira de Composição de Alimentos) 20 ou a tabela oficial de composição nutricional americana 21 , versão 15 , quando o alimento não estava disponível na primeira tabela; (4) soma das calorias provenientes de cada macronutriente de interesse adquiridas pelos domicílios de um mesmo estrato e divisão do resultado pela soma do número de indivíduos no estrato (os fatores amostrais de ponderação de cada domicílio foram utilizados nesta etapa). O fator amostral de ponderação de cada estrato correspondeu à somatória dos fatores amostrais de ponderação dos domicílios pertencentes a cada estrato.

Atributos sócio-demográficos dos estratos de domicílios foram utilizados para caracterizar as unidades de estudo e para controlar possíveis efeitos de confusão para a associação entre calorias adquiridas de açúcar e calorias dos demais macronutrientes. Tais atributos incluíram: a localização (macrorregião Norte, Nordeste, Sudeste, Sul ou Centro-Oeste) e a situação urbana ou rural do estrato de domicílios, além dos valores médios no estrato da renda domiciliar per capita e do percentual de menores de 20 anos, de maiores de 64 anos e de mulheres. Para se chegar a esses últimos valores, foram novamente empregados os fatores amostrais de ponderação de cada domicílio pertencente ao estrato.

\section{Análises estatísticas}

O efeito do açúcar sobre o perfil de macronutrientes na aquisição domiciliar de alimentos foi estudado mediante os coeficientes de modelos de regressão linear múltiplos, específicos para cada macronutriente de interesse utilizando o teste $\mathrm{t}$, adotando-se nível de significância de $\alpha=$ $5 \%$. A variável dependente desses modelos foi o total de calorias correspondente ao macronutriente, e as variáveis explanatórias foram o total de calorias correspondente ao açúcar e ao valor calórico total da aquisição de alimentos, exceto as calorias do açúcar. Com a inclusão da última variável nos modelos, o coeficiente de regressão do açúcar indica a influência que a quantidade consumida de açúcar exerce sobre a quantidade consumida do macronutriente, independentemente de variações no valor calórico total da dieta (excetuadas as calorias do açúcar). Ou seja, nesta situação, o coeficiente de regressão do açúcar indica a participação relativa do macronutriente no valor calórico total da dieta, salvo as calorias do açúcar. Ademais, visando ao controle de potenciais variáveis de confusão para a relação entre aquisição de açúcar e a participação relativa de cada macronutriente na aquisição domiciliar de alimentos, adicionaram-se aos modelos de regressão múltipla as variáveis de caracterização sócio-demográfica das unidades de estudo (estratos de domicílios) que apresentaram valor de $p$ inferior a 0,20 na associação bivariada com o macronutriente de interesse ou com o total de calorias do açúcar. A seguir, utilizando-se o procedimento backward, foram retiradas dos modelos, uma a uma, as potenciais variáveis de confusão, iniciando pela variável com o maior valor de $\mathrm{p}$ e mantendo-se no modelo final todas as variáveis cuja retirada tivesse alterado em mais de $10 \%$ a magnitude do coeficiente de regressão do açúcar. Os pressupostos paramétricos da análise de regressão foram examinados pelos testes de Kolmogorov-Smirnov e Shapiro-Wilk. A adequação dos pressupostos de normalidade e independência dos modelos foi verificada por meio da análise gráfica dos resíduos.

Procedimentos análogos de modelagem foram empregados para se estudar a influência específica do açúcar de mesa (somado a outros adoçantes calóricos empregados pelas famílias, como mel, melado e rapadura) e dos açúcares adicionados pela indústria a alimentos processados.

Em todas as análises deste estudo, foram utilizados fatores amostrais de ponderação que permitem a extrapolação dos resultados para a população brasileira. Todas as análises foram fei- 
tas com o emprego do aplicativo estatístico Stata, versão 8.0 (Stata Corp., College Station, Estados Unidos).

\section{Resultados}

A Tabela 1 mostra que a aquisição de todos os macronutrientes aumenta de modo uniforme e significativo com o aumento das calorias provenientes de açúcar.

A Tabela 2 mostra a associação entre as variáveis sociodemográficas que foram utilizadas para os ajustes dos modelos e as calorias provenientes dos macronutrientes estudados.

A Tabela 3 apresenta os coeficientes dos modelos de regressão relativos à associação entre aquisição domiciliar de açúcar e aquisição de macronutrientes. Os coeficientes de regressão brutos confirmam e quantificam as associações positivas entre açúcar e cada um dos macronutrientes evidenciadas na tabela anterior. Os coeficientes ajustados para o valor calórico total da aquisição de alimentos (excetuadas as calorias do açúcar) indicam, por sua vez, que calorias de açúcar estão associadas a variações significativas na participação dos demais macronutrientes na aquisição domiciliar de alimentos. Cada caloria de açúcar acarreta aumento de 0,39 caloria na participação de gorduras, diminuição de 0,07 caloria na participação de proteínas e de 0,22 caloria dos outros carboidratos que não açúcar.

A Tabela 4 apresenta os coeficientes dos modelos de regressão múltipla relativos às associações entre calorias provenientes do açúcar e de suas frações (açúcar de mesa e açúcar presente em alimentos processados) e calorias provenientes de outros macronutrientes. Esses modelos foram ajustados para o valor calórico total da aquisição de alimentos, tirante as calorias de açúcar (ou de cada fração de açúcar) e, adicionalmente, para as variáveis sociodemográficas que se mostraram variáveis de confusão para a associação entre calorias de açúcar (ou de suas frações) e calorias de cada macronutriente.

Com o ajuste adicional para variáveis sóciodemográficas, os coeficientes de regressão indicam que cada caloria proveniente de açúcar acarreta aumento significativo na participação relativa de 0,3 caloria de gorduras e diminuição de 0,07 caloria, também significativa na participação de proteínas.

Os coeficientes dos modelos de regressão relativos à influência das frações do açúcar sobre a participação relativa na aquisição domiciliar de alimentos dos demais macronutrientes indicam efeitos significativos apenas no caso do açúcar presente em alimentos processados. Cada caloria de açúcar presente em alimentos processados acarreta aumento significativo de 1,6 caloria na participação de gorduras totais e 0,4 caloria de ácidos graxos saturados e diminuição, também significativa, de 0,8 caloria na participação de carboidratos que não o açúcar.

Modelos independentes para situações distintas de renda foram testados, mas não apresentados, em razão de evidenciarem resultados semelhantes aos dados para a média do total dos domicílios.

\section{Discussão}

Neste estudo se evidenciou que aumentos nas calorias adquiridas de açúcar se associam a uma maior participação de gorduras e menor participação de proteínas na aquisição domiciliar de alimentos. Com relação especificamente a aumentos nas calorias da fração de açúcar proveniente de alimentos processados, constatou-se maior participação de gorduras e de ácidos graxos saturados e menor participação de carboidratos que não o açúcar.

As principais limitações a serem consideradas quando se utilizam dados de aquisição domiciliar de alimentos para avaliação do consumo alimentar das famílias são: (i) a não-consideração dos alimentos consumidos fora do domicílio; (ii) o desconhecimento da eventual participação de não moradores nas refeições feitas no domicílio; (iii) a proporção de alimentos adquiridos e não consumidos (“desperdícios"); e (iv) a não-consideração do estoque inicial e final de alimentos nos domicílios estudados. No caso da POF 20022003, soma-se a essas limitações o curto período (uma semana) de registro das aquisições de alimentos em cada domicílio.

A primeira limitação - a não-consideração dos alimentos consumidos fora de casa - deve ter impacto limitado sobre os resultados do presente estudo, uma vez que seu objetivo primordial não é estimar quantidades absolutas de alimentos consumidos pelos indivíduos, mas sim analisar relações entre o consumo de um determinado item da alimentação (açúcar de mesa ou açúcar adicionado a alimentos processados) e o perfil de macronutrientes da dieta. De qualquer forma, análises realizadas separadamente para estratos de domicílios onde se estima que a alimentação fora de casa seja pouco freqüente (estratos cujo gasto com alimentação fora do domicílio era inferior a $10 \%$ do gasto total com alimentação) revelam resultados semelhantes aos obtidos para o conjunto de estratos (resultados não mostrados). 
Tabela 1

Valores médios para calorias provenientes de diferentes macronutrientes segundo quartis das calorias provenientes de açúcar na aquisição domiciliar de alimentos. Brasil, 2002-2003.

\begin{tabular}{|c|c|c|c|c|}
\hline $\begin{array}{l}\text { Quartos da aquisição de açúcar } \\
\text { (kcal/per capita/dia) }\end{array}$ & $\begin{array}{c}\text { Proteína* } \\
\text { (kcal/per capita/dia) }\end{array}$ & $\begin{array}{l}\text { Carboidratos exceto } \\
\text { açúcar refinado * } \\
\text { (kcal/per capita/dia) }\end{array}$ & $\begin{array}{l}\text { Gorduras totais * } \\
\text { (kcal/per capita/dia) }\end{array}$ & $\begin{array}{l}\text { Ácidos graxos saturados * } \\
\text { (kcal/per capita/dia) }\end{array}$ \\
\hline $1 ㅇ(9,19-231,32)$ & 181,87 & 641,62 & 414,95 & 119,19 \\
\hline 2ㅇ $(231,32-290,71)$ & 200,17 & 755,03 & 489,02 & 135,55 \\
\hline 3으 $(292,01-334,82)$ & 218,95 & 806,06 & 546,41 & 152,87 \\
\hline 4으 $(334,83-768,17)$ & 249,90 & 992,13 & 635,15 & 172,60 \\
\hline
\end{tabular}

* Valor de $p<0,000$ para tendência linear.

Tabela 2

Médias de calorias provenientes de açúcar e de calorias provenientes de outros macronutrientes na aquisição domiciliar de alimentos (kcal/per capita/dia) segundo variáveis sócio-demográficas em domicílios brasileiros (2002-2003).

\begin{tabular}{|c|c|c|c|c|}
\hline Variáveis & Gorduras & Proteínas & $\begin{array}{c}\text { Carboidratos exceto } \\
\text { açúcar }\end{array}$ & Gordura saturada \\
\hline \multicolumn{5}{|l|}{ Região } \\
\hline Norte & 496,96 & 232,85 & 937,38 & 141,45 \\
\hline Nordeste & 423,68 & 201,37 & 876,92 & 116,70 \\
\hline Sudeste & 545,41 & 205,92 & 737,29 & 151,30 \\
\hline Sul & 606,72 & 250,83 & 817,83 & 175,50 \\
\hline Centro-Oeste & 536,58 & 189,52 & 730,60 & 138,39 \\
\hline \multicolumn{5}{|l|}{ Área } \\
\hline Urbana & 509,50 * & 202,36 * & 728,38 * & 142,27 \\
\hline Rural & 583,36 & 269,03 & 1183,98 & 159,43 \\
\hline \multicolumn{5}{|c|}{ Renda domiciliar per capita } \\
\hline 1o quarto & 433,73 * & 202,29 & 910,13 * & 114,63 * \\
\hline 2o quarto & 565,98 & 228,34 & 876,35 & 152,78 \\
\hline 3o quarto & 536,28 & 206,80 & 719,64 & 147,47 \\
\hline 4o quarto & 548,73 & 213,12 & 683,04 & 165,77 \\
\hline \multicolumn{5}{|c|}{ Menores de 20 anos (\%) } \\
\hline 1ㅇ quarto & 596,04 * & 231,69 * & 754,20 * & 177,68 * \\
\hline 2o quarto & 536,18 & 212,73 & 747,76 & 148,74 \\
\hline 3o quarto & 505,49 & 198,97 & 778,83 & 134,14 \\
\hline 4o quarto & 444,43 & 206,59 & 911,56 & 118,64 \\
\hline \multicolumn{5}{|c|}{ Maiores de 64 anos (\%) } \\
\hline 1ㅇ quarto & 463,24 * & 192,24 * & 697,49 * & 127,93 * \\
\hline 2o quarto & 493,30 & 203,77 & 820,29 & 135,20 \\
\hline 3o quarto & 535,80 & 216,77 & 857,90 & 143,84 \\
\hline 4o quarto & 592,04 & 237,80 & 816,51 & 173,09 \\
\hline \multicolumn{5}{|l|}{ Mulheres (\%) } \\
\hline 1ㅇ quarto & 541,50 & 236,54 * & 985,82 * & 148,02 \\
\hline 2o quarto & 511,37 & 204,75 & 759,60 & 138,88 \\
\hline 3o quarto & 522,12 & 203,34 & 740,59 & 142,75 \\
\hline 4o quarto & 508,12 & 205,05 & 701,03 & 150,15 \\
\hline Total & 520,76 & 212,52 & 797,86 & 144,88 \\
\hline
\end{tabular}

* Valor de p significativo < 0,05 para tendência linear. 
Tabela 3

Coeficientes de regressão das calorias provenientes de açúcar sobre as calorias provenientes de outros macronutrientes na aquisição domiciliar de alimentos. Brasil, 2002-2003.

\begin{tabular}{lccc}
\hline $\begin{array}{l}\text { Variável dependente no modelo } \\
\text { (kcal/per capita/dia) }\end{array}$ & $\begin{array}{c}\text { Coeficientes brutos } \\
\text { (kcal/per capita/dia) }\end{array}$ & $\begin{array}{c}\text { Coeficientes ajustados * } \\
\text { (kcal/per capita/dia) }\end{array}$ & IC95\% dos coeficientes ajustados \\
\hline Gorduras & 1,059 & 0,393 & 0,208 \\
Ácidos graxos saturados & 0,265 & 0,038 & $-0,030$ \\
Carboidratos exceto açúcar & 1,729 & $-0,224$ & $-0,442$ \\
Proteínas & 0,352 & $-0,067$ & $-0,122$ \\
\hline
\end{tabular}

IC95\%: intervalo de 95\% de confiança.

* Ajustados para o total de calorias adquiridas excetuadas as do açúcar.

Tabela 4

Coeficientes de regressão ajustados das calorias provenientes de açúcar e suas frações sobre as calorias provenientes de outros macronutrientes na aquisição domiciliar de alimentos. Brasil, 2002-2003.

\begin{tabular}{|c|c|c|c|}
\hline \multirow[t]{2}{*}{ Variável dependente do modelo } & Açúcar (kcal/per capita/dia) & $\begin{array}{c}\text { Fração açúcar de mesa (kcal/per } \\
\text { capita/dia) }\end{array}$ & $\begin{array}{c}\text { Fração açúcar presente em } \\
\text { alimentos processados (kcal/per } \\
\text { capita/dia) }\end{array}$ \\
\hline & Coeficiente (IC95\%) & Coeficiente (IC95\%) & Coeficiente (IC95\%) \\
\hline Gorduras totais & $0,313 *(0,158-0,469)$ & $0,177 *(-0,006-0,361)$ & $1,568 * \star(1,241-1,895)$ \\
\hline Ácidos graxos saturados & 0,022 *** $(-0,032-0,075)$ & $-0,019 * * \star(-0,074-0,036)$ & $0,436 \#(0,303-0,570)$ \\
\hline Carboidratos exceto açúcar & $-0,155 \# \#(-0,319-0,010)$ & 0,044 \#\#\# $(-0,165-0,254)$ & $-0,813$ \#\#\# (-1,315;-0,310) \\
\hline Proteínas & $-0,067 \S(-0,122-0,011)$ & $-0,054 \S \S(-0,114-0,006)$ & $0,125 \# \# \#(-0,083-0,334)$ \\
\hline
\end{tabular}

IC95\%: intervalo de 95\% de confiança.

Nota: todos os coeficientes estão ajustados para o total de calorias adquiridas excetuadas as do açúcar.

* Ajuste adicional para região e renda;

** Ajuste adicional para o percentual de menores de 20 anos;

*** Ajuste adicional para região, renda e percentual de menores de 20 anos;

\# Ajuste adicional para renda;

\#\# Ajuste adicional para renda e percentual de menores de 20 anos;

\#\#\# Ajuste adicional para área, região, renda e percentual de menores de 20 anos;

$\S$ Ajuste adicional para região e percentual de menores de 20 anos;

$\S \S$ Ajuste adicional para área, região e percentual de menores de 20 anos.

Quanto à eventual participação de nãomoradores nas refeições feitas nos domicílios e quanto a eventuais desperdícios, novamente o impacto sobre os resultados do nosso estudo deve ser limitado em função de que ele focaliza relações entre diferentes itens da alimentação e não quantidades absolutas de alimentos consumidos.

A não-consideração do estoque inicial e final de alimentos nos domicílios estudados poderia afetar substancialmente os resultados do nosso estudo caso as unidades de análise fossem domicílios individuais. Como as unidades são agre- gadas de domicílios (dezenas ou centenas de domicílios, na maior parte das vezes), variações existentes em cada domicílio entre o estoque de alimentos no início e no final do período de observação tendem a se anular. A mesma argumentação se aplica ao curto período de registro das aquisições de alimentos feitas por cada domicílio individual.

Apesar das limitações características das POF, dados sobre aquisições de alimentos fornecem informações com considerável concordância quando comparadas aos resultados obtidos por meio de inquéritos individuais de consumo, so- 
bretudo quando os indicadores utilizados focam medidas relativas e não absolutas 22,23.

Dois pontos fortes do presente estudo são a representatividade nacional da amostra de domicílios estudada e o controle estatístico de diversos atributos sociodemográficos que poderiam atuar como fator de confusão para a associação entre calorias de açúcar e perfil de macronutrientes da aquisição domiciliar de alimentos. Ainda assim, com relação a este último aspecto, como em qualquer estudo observacional, não se pode descartar a possibilidade de existência de fatores de confusão não considerados em nossa análise.

São escassos os estudos publicados sobre a influência do açúcar na composição da dieta em macronutrientes. Dois estudos realizados com crianças americanas encontraram associação inversa entre consumo de açúcar e consumo de proteínas, de gorduras totais e de ácidos graxos saturados. Com relação à associação entre açúcar e carboidratos totais (inclusive o açúcar), os resultados encontrados nos dois estudos divergem entre si, tendo sido observada associação direta em um deles e inversa no outro 14,17. Em nenhum dos dois estudos houve controle de variáveis sociodemográficas. Além disso, os dois estudos analisam a associação entre açúcar e demais macronutrientes, focando a participação relativa deles no valor calórico total da dieta, procedimento que fere o princípio de independência entre as variáveis estudadas 24 .

Um terceiro estudo realizado na Alemanha com crianças e adolescentes identificou associação inversa entre consumo de açúcar e um escore de alimentação saudável, composto, entre outras variáveis, pelo percentual de participação de gorduras e de ácidos graxos saturados. Pelos resultados deste estudo os autores sugerem como recomendação para a população de crianças e adolescentes alemãs limites entre 6\% e 12\% para a participação do açúcar no valor calórico total da dieta 18 .
A relação empírica encontrada neste estudo entre açúcar proveniente de alimentos processados e participação de gorduras totais e ácidos graxos saturados na disponibilidade domiciliar de alimentos poderia dever-se a dois mecanismos. O primeiro mecanismo seria a presença simultânea (em grande proporção) de açúcar e gorduras nos alimentos processados. Esse parece ser o caso de sorvetes, chocolates e biscoitos doces, produtos que representam $31,7 \%$ do total das calorias de açúcar nos alimentos processados adquiridos pelas famílias brasileiras. Em sorvetes e chocolates há entre $35 \%$ e $39 \%$ de calorias de açúcar e entre $48 \%$ e $51 \%$ de calorias de gorduras (sendo cerca de $60 \%$ provenientes de ácidos graxos saturados). Em biscoitos doces há cerca de $35 \%$ de calorias de açúcar e $25 \%$ de calorias de gorduras.

O segundo mecanismo para a associação entre açúcar proveniente de alimentos processados e participação de gorduras na aquisição domiciliar de alimentos envolveria eventuais associações entre aquisição de alimentos processados ricos em açúcar e aquisição de alimentos ricos em gorduras. A correlação positiva entre aquisição de refrigerantes $(42,9 \%$ do total das calorias de açúcar nos alimentos processados adquiridos pelas famílias brasileiras) e a aquisição de doces, biscoitos e óleos e gorduras exemplifica bem este segundo mecanismo (coeficientes de correlação parcial, com ajuste para calorias totais, de 0,70 para doces, 0,28 para biscoitos e 0,24 para óleos e gorduras; todos com $\mathrm{p}<0,001$ ) (dados não mostrados).

Independentemente dos mecanismos pelos quais a aquisição de açúcar (em geral e açúcar proveniente de alimentos processados) influencia o perfil de macronutriente da aquisição domiciliar de alimentos das famílias brasileiras, os resultados mostrados neste estudo trazem novas evidências sobre o papel prejudicial do açúcar à saúde humana. Essas evidências corroboram orientações brasileiras 25 e internacionais 7 , que recomendam a redução no consumo de açúcar e estipulam limites máximos para sua participação na dieta. 


\section{Resumo}

Avaliou-se a influência exercida pela aquisição de calorias de açúcar sobre a participação calórica dos demais macronutrientes da dieta. As estimativas deste estudo são baseadas em dados da Pesquisa de Orçamentos Familiares realizada no Brasil pelo Instituto Brasileiro de Geografia e Estatística entre julho de 2002 e junho de 2003. Modelos de regressão linear múltiplos foram utilizados para estudar a influência das calorias de açúcar sobre a participação calórica de cada um dos macronutrientes na aquisição domiciliar de alimentos com o controle do valor calórico total da aquisição de alimentos e variáveis sócio-demográficas. Cada caloria adquirida de açúcar eleva em 0,3 caloria a participação de gorduras na aquisição domiciliar de alimentos e diminui em 0,07 a participação de proteinas. Cada caloria de açúcar procedente de alimentos processados aumenta em 1,6 caloria a participação de gorduras e em 0,4 caloria de ácidos graxos saturados $e$ diminui em 0,8 caloria a participação de outros carboidratos que não o açúcar. Os resultados encontrados trazem novas evidências sobre o papel prejudicial do açúcar à saúde humana.

Comportamento Alimentar; Açúcar; Ingestão de Alimentos

\section{Referências}

1. Popkin B, Doak CM. The obesity epidemic is a worldwide phenomenon. Nutr Rev 1998; 56: 106-14.

2. Instituto Brasileiro de Geografia e Estatística. Pesquisa de Orçamentos Familiares 2002-2003: análise da disponibilidade domiciliar de alimentos e do estado nutricional no Brasil. Rio de Janeiro: Instituto Brasileiro de Geografia e Estatística; 2004.

3. Instituto Brasileiro de Geografia e Estatística. Pesquisa de Orçamentos Familiares 2002-2003: antropometria e análise do nutricional de crianças e adolescentes no Brasil. Rio de Janeiro: Instituto Brasileiro de Geografia e Estatística; 2006.

4. Monteiro CA, Conde WL, Popkin BM. Incomespecific shifts in obesity in Brazil: 1975-2003. Am J Public Health 2007; 97:1808-12.

\section{Colaboradores}

R. B. Levy contribuiu no planejamento do estudo, análise dos dados e na redação do artigo. R. M. Claro colaborou na análise de dados e redação do artigo. C. A. Monteiro participou do planejamento do estudo e redação do artigo.
5. Popkin BM. The shift in stages of the nutrition transition in the developing world differs from past experiences! Public Health Nutr 2002; 5(1A): 205-14.

6. Bermudez OI, Tucker KL. Trends in dietary patterns of Latin American populations. Cad Saúde Pública 2003; 19 Suppl 1:S87-99.

7. World Health Organization/Food and Agriculture Organization. Diet, nutrition and the prevention of chronic diseases. Geneva: World Health Organization; 2003. (WHO Technical Report Series, 916).

8. Institute of Medicine. Dietary reference intakes for energy, carbohydrate, fiber, fat, fatty acids, cholesterol, protein, and amino acids. Washington DC: National Academies Press; 2002. 
9. Ludwig DS, Peterson KE, Gortmaker SL. Relation between consumption of sugar-sweetened drinks and childhood obesity: a prospective, observational analysis. Lancet 2001; 357:505-8.

10. Dubois L, Farmer A, Girard M, Peterson K. Regular sugar-sweetened beverage consumption between meals increases risk of overweight among preschool-aged children. J Am Diet Assoc 2007; 107:924-34.

11. Fried SK, Rao SP. Sugars, hypertriglyceridemia and cardiovascular disease. Am J Clin Nutr 2003; 78:873S-80S.

12. Kelley DE. Sugar and starch in the nutritional management of diabetes mellitus. Am J Clin Nutr 2003; 78:858S-64S

13. Levy RB, Claro RM, Monteiro CA. Sugar and total energy content of household food purchases in Brazil. Public Health Nutr 2009; 12:2084-91.

14. Kranz S, Smiciklas-Wright H, Siega-Riz AM, Mitchell D. Adverse affect of high added sugar consumption on diet intake in American preschoolers. J Pediatr 2005; 146:105-11.

15. Popkin BM, Nielsen SJ. The sweetening of the world's diet. Obes Res 2003; 11:1325-32.

16. Mann J. Free sugars and human health: sufficient evidence for action? Lancet 2004; 363:1068-70.

17. Farris RP, Nicklas TA, Myers L, Berenson GS. Nutrient intake and food group consumption of 10 year-olds by sugar intake level: the Bogalusa Heart Study. J Am Coll Nutr 1998; 17:579-85.

18. Ute A, Wolfgang SH, Kersting M. Fortification masks nutrient dilution due to added sugars in the diet of children and adolescents. J Nutr 2002; 132:2785-91.
19. Instituto Brasileiro de Geografia e Estatística. Estudo Nacional da Despesa Familiar: tabela de composição de alimentos. 4a Ed. Rio de Janeiro: Instituto Brasileiro de Geografia e Estatística; 1996.

20. Núcleo de Estudos e Pesquisas em Alimentação, Universidade Estadual de Campinas. Tabela Brasileira de Composição de Alimentos - TACO. Versão 1. Campinas: Núcleo de Estudos e Pesquisas em Alimentação, Universidade Estadual de Campinas; 2004.

21. United States Department of Agriculture. USDA Food Search for Windows, version 1.0, database version Standard Reference Release SR16. Agricultural Research Service (ARS). http://www.nal.usda. gov/fnic/foodcomp (acessado em Mar/2006).

22. Naska A, Vasdekis VGS, Trichopoulou A. A preliminary assessment of the use of household budget survey data for the prediction of individual food consumption. Public Health Nutr 2001; 4(5B):1159-65.

23. Becker W. Comparability of household and individual food consumption data - evidence from Sweden. Public Health Nutr 2001; 4(5B):1177-82.

24. Forshee RA, Storey ML. Controversy and statistical issues in the use of nutrient densities in assessing diet quality. J Nutr 2004; 134:2733-7.

25. Coordenação-Geral da Política de Alimentação e Nutrição, Secretaria de Atenção à Saúde, Ministério da Saúde. Guia alimentar para a população brasileira: promovendo a alimentação saudável. Brasília: Ministério da Saúde; 2006.

Recebido em 31/Mar/2009

Versão final reapresentada em 07/Jan/2010 Aprovado em 05/Fev/2010 